\title{
Caracterización Socioeconómica, Ambiental y de género en los sistemas de producción de las microcuencas Ríos Cristal y el Salto - Provincias Bolívar y los Ríos, Ecuador
}

\section{Socioeconomic characterization, environment and Gender in the production systems of the microcuences Rio Cristal and the Salto - Provinces Bolívar and the Ríos, Ecuador}

\author{
Martha González ${ }^{1}$ \\ marthy_1@yahoo.es \\ Nelson Monar ${ }^{1}$ \\ Sonia Fierro ${ }^{1}$ \\ KleberEspinoza ${ }^{1}$ \\ Guillermo Vizcarra1 \\ Rubén Saltos ${ }^{2}$ \\ rudasaes@yahoo.es \\ ${ }^{1}$ Escuela de Ingeniería forestal de la Universidad Estatal de Bolívar \\ ${ }^{2}$ Ministerio de Agricultura Ganadería Acuacultura y Pesca
}

\begin{abstract}
RESUMEN
Este estudio permitió la sistematización y verificación efectiva, de la problemática y potencialidades de las microcuencas del rio el Salto y el Cristal para buscar soluciones sostenibles. El objetivo fue caracterizar socioeconómica y ambientalmente los sistemas de producción de las microcuencas del rio Cristal y el Salto de las provincias Bolívar y de Los Ríos en Ecuador. Se usó la metodología del paradigma de investigación socio-crítico, fundamentado en la ciencia de investigación-acción. Se usaron estrategias metodológicas de investigación-acción participativa. Los Resultados obtenidos fue la caracterización socioeconómica y ambiental de los sistemas de producción. Las microcuencas de los ríos Cristal y El Salto, se recopiló la información de 68 familias, luego se analizó y se sistematizó la información que permitió caracterizar los sistemas de producción. Para el análisis de cuencas hidrográficas se requiere de una extensa información de cartografía digitalizada misma que es muy limitada en zonas de alta nubosidad.
\end{abstract}

Palabras clave: Caracterización socioeconómica; sistematización; microcuencas

\begin{abstract}
This study allowed the systematization and effective verification, of the problematic and potentialities of the microwatersheds of the river the Salto and the Crystal to look for sustainable solutions. The objective was to characterize socioeconomically and environmentally the systems of production of the microwatersheds of the Crystal River and the Salto of the provinces Bolivar and of The Rivers in Ecuador. The methodology of the sociocritical research paradigm, based on research-action science, was used. Methodological participatory Action research strategies were used. The Results obtained were the socio-economic and environmental characterization of the production systems. The microwatersheds of the Cristal Rivers and The Salto, the information of 68 families was compiled, then the information that allowed to characterize the production systems was analyzed and systematized. For the analysis of watersheds it is necessary to have extensive information on digitized cartography itself that is very limited in high cloud areas.
\end{abstract}

Key words: Socioeconomic characterization; systematization; microwatersheds 


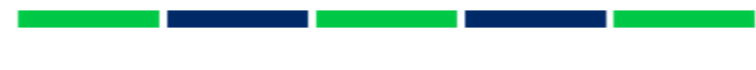

INTRODUCCIÓN

Las Microcuencas del río Cristal y el Salto, situado en los cantones de San Miguel (Provincia de Bolívar) y Montalvo (provincia de Los Ríos). Compuesta por $1050 \mathrm{Ha}$, presentan un caudal en el río El Salto de 2.000 lt./s. y el rio Cristal de 150 lt./s., de los cuales se desprenden vertientes y tomas de agua consideradas como las principales sistemas de abastecimiento para los cantones San Miguel y Montalvo (VI Foro Nacional Climático, 2011).

El proyecto "Manejo Integrado de los Recursos Naturales para agricultura de Pequeña Escala con base a Cuencas Hidrográficas: El caso de la Microcuenca del Rio Cristal y El Salto de las provincias Bolívar y los Ríos - Ecuador", orienta sus labores en el manejo integrado de los recursos naturales utilizando el enfoque de Cuencas Hidrográficas, desde la equidad socioeconómica, ambiental y de género con la participación activa de entes gubernamentales y ONGs.

La Universidad Estatal de Bolívar y la Facultad de Ciencias Agropecuarias, Recursos Naturales y del Ambiente a través de la Escuela de Ingeniería Forestal contribuyen al desarrollo sostenible de la microcuencas hidrográficas del río Cristal y el Salto, mediante el manejo integrado de los recursos naturales para agricultura de pequeña escala, con equidad ambiental, social y de género, para ello consideró urgente realizar el estudio de Línea Base, para determinar potencialidades y limitantes de los sistemas de producción desde una perspectiva de las actividades de sustento; en las microcuencas a través de la caracterización de los sistemas de producción y de los recursos naturales que contribuirá a la seguridad alimentaria y reducción de la pobreza de las familias.

La sistematización de éste estudio ha permitido contribuir a una verificación efectiva, de la problemática y potencialidades de las microcuencas del rio el Salto y el río Cristal para buscar soluciones sostenibles a los principales problemas.

\section{Objetivos}

\section{Objetivo general}

Caracterizar Socioeconómica y Ambiental de los Sistemas de Producción de las Microcuencas del Rio Cristal y el Salto de Las Provincias Bolívar y de Los Ríos - Ecuador.

\section{Objetivos específicos}

- Recopilar, sistematizar y analizar información que contribuya al mejoramiento de los sistemas de producción localizados en las Microcuencas de los Ríos Cristal y el Salta.

- Identificar los principales factores limitantes y potencialidades de los Sistemas de Producción en las Microcuencas de los Ríos Cristal y el Salto.

- Describir la composición socioeconómica de las familias de las Microcuencas de los Ríos Cristal y el Salto.

\section{MATERIALES Y MÉTODOS}

El estudio se enmarcó en las metodologías del paradigma de investigación socio-crítico, fundamentado en la ciencia de investigación-acción. Se utilizaron varias estrategias metodológicas de la investigaciónacción participativa IAP (Barton, 1997; INIAP, 2001; citado por González, M. 2008) y de análisis de género (CIEG, 2003; INIAP, 2001; citado Cárdenas, F. Barrera, 2006). Se realizó un tipo de estudio exploratorio y descriptivo, con base en el análisis de la situación sociocultural, económica productiva, ambiental e institucional-político de los territorios en estudio. 
Se integró los datos secundarios (revisión de información secundaria relacionada con el manejo de recursos naturales en las Microcuencas, mapas, bases de datos socioeconómicas y agroecológicas, entre otros) con datos primarios, generados con la aplicación de la caracterización socioeconómica y ambiental con enfoque de género (CSAEG), en las comunidades de las Microcuencas del río Cristal y El Salto distribuidas en dos partes: 1) Parte alta: Santa Lucia y San Cristóbal, El Alcacer, San Vicente 2) Parte media: El cristal, Balsapamba Central, Chaupiyacu, La Plancha y las Peñas.

\section{Zona de vida}

Las microcuencas se encuentran en la zona Premontano o Subtropical se extiende desde los 300 hasta $2000 \mathrm{msnm}$ con temperaturas de 18 a $24{ }^{\circ} \mathrm{C}$ y precipitaciones entre 1000 y $3000 \mathrm{~mm}$ anuales. Comprende las áreas de Facundo Vela, Balsapamba, Telimbela, San José del Tambo, Caluma, Echeandia y las Naves. Cubre aproximadamente $644 \mathrm{~km} 2$ (16.41\%) de la superficie total.

\section{Clasificación taxonómica del suelo}

Los suelos de la provincia Bolívar corresponden a un marco geológico regional muy complejo, de composición litológica diversa que ha dado origen a una variedad de tipos de suelos, cuya composición físicaquímica y textura está caracterizada por los factores climáticos y por el relieve que presenta. En el cuadro No 1. Se muestra los tipos de suelo que existen en las microcuencas:

Cuadro 1. Clasificación taxonómica del suelo de las Microcuencas del río Cristal y el Salto, provincia de Bolívar y los Ríos, Ecuador.

\begin{tabular}{clcl}
\hline $\begin{array}{c}\text { Clasificación } \\
\text { taxonómica }\end{array}$ & \multicolumn{1}{c}{ Características } & Altura & \multicolumn{1}{c}{ Localización } \\
\hline $\begin{array}{c}\text { Eutropets y/o } \\
\text { Tropudales }\end{array}$ & $\begin{array}{l}\text { Suelos pardo rojizos, arcillo } \\
\text { arenosos, medianos o poco } \\
\text { profundos, algo rocosos, } \\
\text { pH 5.5-6.5. }\end{array}$ & $\begin{array}{l}300- \\
1500\end{array}$ & $\begin{array}{l}\text { Límite occidental y } \\
\text { sur de Bolívar }\end{array}$ \\
& $\begin{array}{l}\text { Suelos rojos, arcillosos o } \\
\text { arcillo arenosos, profundos, } \\
\text { pedregosos, pH 4-5.5. }\end{array}$ & $1500-$ & $\begin{array}{l}\text { Extremo sur de } \\
\text { Bolívar. } \\
\text { Pystropepts }\end{array}$ \\
& \multicolumn{1}{c}{$\begin{array}{l}\text { Parte inferior de } \\
\text { las vertientes } \\
\text { occidentales. }\end{array}$} \\
\hline Tropofluvents & $\begin{array}{l}\text { Suelos pluviales, textura } \\
\text { variable, limosos o } \\
\text { arcillosos, profundos, } \\
\text { pedregosos. }\end{array}$ & 2400 & $\begin{array}{l}\text { Terrazas medias } \\
\text { de los ríos del } \\
\text { centro y occidente } \\
\text { de Bolívar }\end{array}$ \\
\hline
\end{tabular}

Fuente: Plan Estratégico de Desarrollo Provincial de Bolívar, 2004-2024.

RESULTADOS Y DISCUSIÓN

La caracterización socioeconómica y ambiental de los sistemas de producción se realizó en dos Microcuencas del cantón San Miguel: la Microcuenca del rio Cristal y la

Alfa, Revista de Investigación en Ciencias Agronómicas y Veterinarias Vol. 1, N No. 2, Mayo-Agosto-2017 
Microcuenca del rio el Salto afluentes de la Subcuenca del río Catarama.

\section{Ámbito Socio-Cultural}

En cuanto a la etnia, los habitantes de las Microcuencas del rio Cristal y el Salto se consideran mestizas el $100 \%$. Las familias de los encuestados el $81,9 \%$ pertenecen a la del rio Cristal y $18,1 \%$ al del Salto; están compuestas por tres miembros el $23,53 \%$, cuatro miembros $17,65 \%$, cinco miembros $16,18 \%$, seis y siete miembros el $8,82 \%$ respectivamente, un miembro el 7,35\% y ocho y nueve miembros el $2,94 \%$ respectivamente, estas familias están conformadas en su mayoría por esposa/so e hijos/as.

En las microcuencas la mayoría de la población de niños/as (aproximadamente 80,9\%) tiene acceso a la educación básica; al nivel secundario acceden el $36,9 \%$ de la población de jóvenes hombres y mujeres y algunos a la Universidad.

Las actividades productivas principales de los habitantes de las microcuencas son: estudiantes en un 35,8\%; agricultura el 23,4\%; quehaceres domésticos en 19,9\%; у а otras actividades en un porcentaje menor 20,9\%; y las actividades secundarias a la que se dedican son a la agricultura 38,3\%; estudiante en un 22,3\%; quehaceres domésticos en un 14,9\% y a otras actividades el $24,5 \%$.

\section{Tenencia y uso del Suelo}

La tenencia de la tierra en las microcuencas se encuentra distribuidas en: propio con título un $71,9 \%$, y sin título un 12,4 $\%$, arrendado un $4,5 \%$ y cedido - prestado el $11,2 \%$. La topografía de los terrenos en las microcuencas, en su mayoría, es ondulada $62,9 \%$, seguida de la topografía plana $21,3 \%$, y quebrada $15,7 \%$. El mismo comportamiento de las microcuencas se reporta a nivel de la subcuenca.

Las microcuencas en estudio ocupan una superficie de 1050 ha $(10,49 \mathrm{Km} 2)$. En las microcuencas del río Cristal y el Salto, la superficie por familia que poseen es menor a 5ha que equivale al $87,5 \%$ de la población encuestada; de 5,1 \pm 10 ha son un $4,5 \%$ y finalmente mayores a 10,1 ha son el $8 \%$; siendo el gran total de 269ha.

Las comunidades de las microcuencas, se caracterizan por ser agrícola y pecuaria. Esta situación se da debido a que la superficie sembrada de cultivos de secano se encuentra en las microcuencas distribuida en un $31 \%$ en el del río El Salto y el 69\% en el río Cristal. Un caso interesante en esta microcuenca es que un alto porcentaje $34,8 \%$ de terrenos se encuentran como matorrales muchas de las veces por encontrarse abandonados o solo con un cuidador para los animales (bovinos) cabe destacar que estos territorios no cuentan con un sistema de riego.

Actualmente en las microcuencas los cultivos están distribuidos de la siguiente forma: 
Cuadro 2. Cultivos actuales (Ha) en las microcuencas del Río Cristal y el Salto, provincia de Bolívar, Ecuador.

\begin{tabular}{lcc}
\hline \multicolumn{1}{c}{ Cultivos } & Superficie Ha & $\mathbf{0}$ \\
\hline $\begin{array}{l}\text { Pasto/caña de azúcar/plátano/papa } \\
\text { china }\end{array}$ & 95,01 & 41,9 \\
Guineo/naranja/orito & 36,44 & 16,1 \\
\hline Café/guineo/naranja & 28,47 & 12,6 \\
Cacao/plátano/yuca & 22,81 & 10,1 \\
Caña de azúcar/papa china/plátano & 13,45 & 5,9 \\
Balsa & 11,36 & 5,0 \\
Naranja/guineo/laurel & 9,64 & 4,3 \\
Plátano/naranja/ caco & 6,36 & 2,8 \\
Maíz amarillo & 2,65 & 1,2 \\
Papaya/naranja/limón & 0,30 & 0,1 \\
\hline \multicolumn{1}{c}{ Total } & $\mathbf{2 2 6 , 4 9 h a}$ & $\mathbf{1 0 0}$ \\
\hline
\end{tabular}

\section{Producción}

Analizando los rendimientos de producción de los diferentes cultivos de mostro que predominan las actividades pecuarias en la parte alta y agrícola en la parte media donde los rubros de mayor rédito económico corresponden a los sistemas maíz/pasto y Orito/seda, además de otros cultivos de ciclo corto y perenne que pueden ser observar en el Cuadro No3. Haciendo una comparación con datos de rendimiento de los diferentes cultivos reportados por en INEC podemos acotar que la productividad de la zona en estudio son relativamente bajos en la mayoría de sus cultivos.

Cuadro 3. Rendimientos promedios de los principales rubros agrícolas en las microcuencas del Río Cristal y el Salto, provincia de Bolívar, Ecuador.

\begin{tabular}{llrl}
\hline \multicolumn{1}{c}{ Cultivos } & \multicolumn{1}{c}{ Rombre $/$ ha } \\
\multicolumn{1}{c}{$\begin{array}{c}\text { Nombre } \\
\text { Comentífico }\end{array}$} & & \\
\hline Banano & Mussa paradisiaca & 189,00 & Racimas \\
Naranja & Citrus sinensis & $13.291,00$ & Unidades \\
Plátano & Mussa sp. & 123,00 & Racimas \\
Café & Coffe arabica & 350,77 & Kilogramos \\
Cacao & Theobroma cacao & 48,71 & Kilogramos \\
Caña de azúcar & Sacharumo & 227,60 & Kilogramos \\
& fficinarum & & \\
Papa china & Dioscorea trífida & 1570,84 & Kilogramos \\
Maíz amarillo & Zea mayz & 216,77 & Kilogramos \\
Limón/naranja & Citrus limón & $35.821,00$ & Unidades \\
Yuca & Yucca sp. & 195,79 & Kilogramos \\
Maíz suave & Zea mays L. & 615,25 & Kilogramos \\
Papaya & Carica & $3.000,00$ & Unidades \\
& papaya & & \\
\hline
\end{tabular}




\section{Mano de Obra}

Las actividades del predio son realizadas con mano de obra familiar, principalmente. En términos generales, en las microcuencas del río Cristal y El Salto existe disponibilidad de mano de obra, es barata, cuyos valores de 4,90 dólares por jornal (día de 8 horas de trabajo).

La mano de obra contratada y familiar dentro de las microcuencas del río Cristal y El Salto está encaminada principalmente a los cultivos de guineo, naranja, plátano y caña de azúcar, la asociación guineo/naranja, y a la producción animal; sobresaliendo los bovinos para producción doble propósito (carne y leche).

La mano de obra contratada proviene de las mismas comunidades del sector, el jornal día actualmente se cuantifica en USD 4 con alimentación en la microcuenca del río Cristal y el Salto y USD 5 sin alimentación.

El porcentaje de participación de las mujeres según cada cultivo es el siguiente:

En relación a la mano de obra que se utiliza en la producción animal, se debe señalar que los sistemas de producción de las microcuencas en estudio, río Cristal y río el Salto, no contratan mano de obra. Básicamente la mano de obra familiar que se utiliza principalmente para actividades como: alimentación de los animales, pastoreo de los bovinos, desbroce de potreros, ordeño de bovinos y control de animales en potrero en las microcuencas utilizan 227 jornales/año/familia, destinando la mayor cantidad de mano de obra hacia las actividades de pastoreo de bovinos y ordeño. Esto tiene relación con la tradición de los sistemas de producción, que como el caso de la parte alta de las microcuencas, que se caracterizan por la implementación de pasturas mejoradas para la producción de leche y carne de bovinos.

En lo referente a salarios y jornales que los habitantes de las microcuencas perciben mensualmente se pueden señalar que en promedio se encuentra distribuidos así: Jornales agrícolas en el sitio $\$ 133,43$; Jornales agrícolas en otro sitio $\$ 167,92$; Jornales de construcción en otro sitio $\$ 175,00$; Salario de empleo fijo $\$ 619,14$ y Salario a contrato $\$ 178,14$, los mismos que se encuentra representados en porcentaje según el número de encuestados.

\section{Distribución de ingresos y egresos}

Los ingresos de los habitantes de las microcuencas provienen principalmente de actividades agrícolas y pecuarias. El 48.53\% de las familias encuestada de las microcuencas son beneficiarios del bono de desarrollo humano, la producción y comercialización de productos agrícolas representa el 35\%, constituyen los ingresos más importantes para los productores que se dedican a estas actividades y sus familias.

Con relación a los egresos, las familias de las comunidades en estudio indicaron varios tipos de gasto que les significa egresos, pero los principales estaban atribuidos por los gastos realizados para los cultivos, mantenimiento de pastizales y animales que se encuentran en producción. A estos gastos se suman todos aquellos que tienen relación con servicios básicos como: agua, luz, teléfono y también aquellos relacionados con necesidades básicas como: alimentación, vestimenta, educación y salud, principalmente. Estos gastos en las microcuencas de los ríos Cristal y El Salto en promedio ascienden a un total de $\$ 1650$.

- 33,38 dólares anuales.

\section{Análisis de la equidad de género, social y ambiental}

Él estudió deja entrever en las microcuencas, la poca participación de las mujeres respecto a la toma de decisiones para la venta de sus productos, que mayormente la hacen los hombres, aun cuando ellas conocen sus precios de venta. 
En las microcuencas las necesidades básicas son sentidas aproximadamente por igual por todos/as los miembros de la familia, cuya satisfacción no altera los roles y las relaciones tradicionales entre hombres $\mathrm{y}$ mujeres, ni modifican su posición en su comunidad; en cambio, en las necesidades estratégicas de género, se visualizó diferentes connotaciones, porque pueden representar un cambio en la posición, en la división del trabajo y relaciones entre géneros, así como facilitar su acceso a las oportunidades de trabajo.

\section{Análisis del recurso natural y ambiental recursos hídricos}

Los entrevistados/as de las microcuencas manifestaron que el agua para consumo humano lo obtienen en un $48.5 \%$ de vertientes, un $32.4 \%$ de agua entubada y en un menor porcentajes de red pública, rio y/o pozo.
Los entrevistados/as de las microcuencas manifestaron mayoritariamente que el agua para consumo humano llega hasta su domicilio y mencionan que el líquido vital no es tratado, a pesar de ello consideran que es de buena calidad porque hasta la actualidad no se han presentado enfermedades por el consumo de la misma y en un menor porcentaje manifestaron que no es de buena calidad porque se presentaron algunas enfermedades parasitarias que se derivan por el consumo de agua no cocida.

Los entrevistados/as de las microcuencas manifestaron que el caudal de agua ha disminuido en los últimos años según su percepción, desconoce el porqué de la disminución del caudal y un porcentaje menor aluden que según su criterio el remanente de agua sigue igual, finalmente mencionan que el caudal de las vertientes y los ríos ha incrementado en los últimos años por intensidad de lluvia/inviernos.

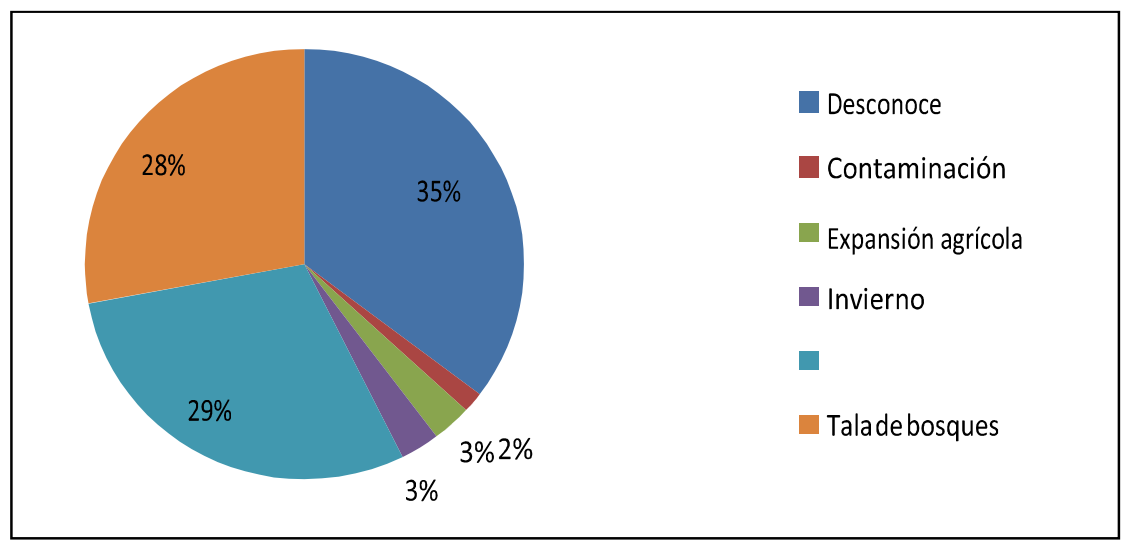

Figura 4. Percepción de los factores que inciden en la variación del caudal de agua en las microcuencas de los ríos Cristal y el Salto

En lo referente a factores que han influenciado en la variación del caudal de los ríos tenemos las antropogénicas y los fenómenos naturales como el verano, la tala indiscriminada de los bosques, la expansión de la frontera agrícola, altos niveles de precipitación en la época de invierno. El 35\% de la población entrevistada manifiestan que desconocen a que se deba la variabilidad del caudal de agua en la zona en estudio.

\section{Protección de vertientes en las microcuencas de los ríos Cristal y el Salto}

La población indica que para proteger las áreas de recarga hídrica, una alternativa es plantando especies nativas y conservando la 
vegetación aún existen dentro de las fuentes y nacientes; y un porcentaje considerable desconoce las alternativas de manejo $\mathrm{y}$ conservación de la flora y fauna.

\section{Análisis del manejo de los recursos:} Bosque y consumo de leña

Según los resultados obtenidos de los entrevistados mencionan que el entorno sigue igual pero un porcentaje de ellos manifiestan que ha disminuido los bosques debido a la tala (desmontes), áreas que son utilizadas a posterior para el establecimiento de pasturas y cultivos; además las especies forestales son aprovechadas para madera y leña entre otros derivados del bosque.

\section{Flora y fauna de las microcuencas del rio Cristal y el Salto}

En lo referente a la flora las especies más relevantes desde el punto de vista económico y resultados obtenidos son: el laurel, Fernán Sánchez, jigua, cascarilla, motilón, y demás especies descritas en el cuadro 4. Cabe mencionar que ciertas especies tanto de flora como fauna se encuentran en peligro de extinción según Acosta Solís.

Cuadro 4. Flora y fauna de Microcuencas de los ríos Cristal y el Salto, provincia Bolívar, Ecuador.

\begin{tabular}{|c|c|c|c|}
\hline \multicolumn{4}{|c|}{ RECURSOS NATURALES } \\
\hline \multicolumn{2}{|l|}{ FLORA } & \multicolumn{2}{|l|}{ FAUNA } \\
\hline $\begin{array}{l}\text { Nombre } \\
\text { Común }\end{array}$ & Nombre Científico & $\begin{array}{l}\text { Nombre } \\
\text { Común }\end{array}$ & Nombre Científico \\
\hline Laurel & Cordia alliodora(R.y P.) & Guatusa & Dasyprocta punctata \\
\hline Fernán & Triplari scumingiana & Guanta & Agoutitae zanowskii \\
\hline Sánchez & & & \\
\hline Quiebracha & Schiopsis quebracho & Armadillo & Dasypus novemincinctus \\
\hline Jigua & Nectandra sp. & Ardilla & Eutimias sibiricus \\
\hline $\begin{array}{l}\text { Sangre de } \\
\text { drago }\end{array}$ & Croton lechleri Muell. & Venado & Hippocamelus antisiensis \\
\hline Cascarilla & Cinchona pubescens & Loro & Amazona farinosa \\
\hline Motilón & Hyeronima asperiflora & Culebra & Suborden: Serpentes \\
\hline Matapalo & Picus paraensis & Carpintero & $\begin{array}{l}\text { Campephilus } \\
\text { magellanicus }\end{array}$ \\
\hline Chilca & Baccharis floribunda & Mono & Ateles geoffroyi \\
\hline Lechero & Euphorbia laurifolia lamb. & Zorro & Vulpes vulpes \\
\hline Cabo de Hacha & Trichiliahirta & Tejón & Meles meles \\
\hline Balsa & Ochroma pyrimidale & Raposa & Metachirus nudicaudatus \\
\hline Orquídeas & Orchidaceae sp. & Pavas & Penelope purpurascens \\
\hline Guabo & Inga vera & Aves & Familia Aludidae \\
\hline Tiumbil & Clusia alata & Sahino & Tayas sutajacu \\
\hline Puma Rosa & Syzygium jambos & Pato de monte & Anas platyrhynchos \\
\hline Moral & Maclura tinctoria & $\begin{array}{l}\text { Oso } \\
\text { hormiguero }\end{array}$ & Tamandua tetradactyla \\
\hline Guayacán & Tabebuia chrysantha & Mirlo & Turdus merula \\
\hline Guarumo & Cecropia litorales & Iguana & Iguana iguana \\
\hline Caucho & Ficus elástica & Gorrión & Passer domesticus \\
\hline Caoba & Swietenia mahagoni L & Cuchucho & Nasua larica \\
\hline Caña Guadua & Guadua angustifolia & Conejos & Oryctola gusciniculus \\
\hline Achiote & Bixa Orellana & Colibríes & Archilochus colubris \\
\hline
\end{tabular}


Uso de especies forestales no maderables

Según los entrevistados/as manifiestan que las plantas medicinales usadas, son unas provenientes de las propiedades y otras son adquiridas en los mercados de la localidad; mismas que detallamos en el siguiente cuadro:

Cuadro 5. Plantas medicinales usadas por los hogares en las Microcuencas de los ríos Cristal y el Salto, provincia Bolívar, Ecuador.

\begin{tabular}{|c|c|c|c|}
\hline DESCRIPCIÓN & $\begin{array}{l}\text { NOMBRE } \\
\text { CIENTÍFICO }\end{array}$ & DESCRIPCIÓN & $\begin{array}{l}\text { NOMBRE } \\
\text { CIENTÍFICO }\end{array}$ \\
\hline Toronjil & Mellisa officinalis & Cola de caballo & $\begin{array}{l}\text { Esquisetum } \\
\text { arvense }\end{array}$ \\
\hline Hierba luisa & $\begin{array}{l}\text { Cimbopogon } \\
\text { citratos }\end{array}$ & Ruda & Ruta graveolens L. \\
\hline Hierba buena & Menta piperita L. & Pumín & Hiptis pecticn \\
\hline Llantén & Plantago major & Violeta & $\begin{array}{l}\text { Sainttpaulia } \\
\text { ionantha } w .\end{array}$ \\
\hline Guabiduca & Piper carpunya & Cilantro & $\begin{array}{l}\text { Coriandrum } \\
\text { sativum }\end{array}$ \\
\hline Manzanilla & $\begin{array}{l}\text { Matricaria } \\
\text { chamomilla }\end{array}$ & Boldo & Peumus boldus \\
\hline Sábila & Aloe vera & Dulcamara & $\begin{array}{l}\text { Solanum } \\
\text { dulcamara }\end{array}$ \\
\hline Albaca & Ocimun bacilicum L. & Chaya & $\begin{array}{l}\text { Cnidos colus } \\
\text { chayamansa }\end{array}$ \\
\hline Menta & Menta piperita & Zaragoza & $\begin{array}{l}\text { Aristolochia } \\
\text { adoratissima L. }\end{array}$ \\
\hline Escancel & $\begin{array}{l}\text { Alternanth } \\
\text { era } \\
\text { pubiflora }\end{array}$ & & \\
\hline
\end{tabular}

Las plantas medicinales son utilizadas como aguas aromáticas, dolores de estómago, inflamaciones, dolor de corazón, cólicos, sistema nervioso y como condimentos en la preparación de alimentos y es consumida por todos los miembros del hogar.

\section{Manejo de los Recursos: Suelo}

En lo concerniente al recurso suelo en las microcuencas, los resultados se muestran a continuación en la gráfica 6 y cuadro 6: 
Cuadro 6. Recurso suelo en las Microcuencas de los ríos Cristal y el Salto, provincia Bolívar, Ecuador.

\begin{tabular}{|c|c|c|c|}
\hline \multicolumn{4}{|c|}{ RECURSO SUELO } \\
\hline \multicolumn{2}{|c|}{ CALIDAD DE LOS SUELOS } & \multicolumn{2}{|c|}{ TOPOGRAFÍA DE LA PROPIEDAD } \\
\hline DESCRIPCIÓN & $\%$ & DESCRIPCIÓN & $\%$ \\
\hline Bueno & 13,2 & Desconoce & 39,7 \\
\hline Regular & 38,3 & Plana & 2,9 \\
\hline Malo & 8,8 & Ondulada & 51,5 \\
\hline desconoce & 39,7 & Quebrada & 5,9 \\
\hline Porcentaje total & 100 & Porcentaje total & 100 \\
\hline \multicolumn{2}{|l|}{ POR QUÉ } & \multicolumn{2}{|c|}{ PRACTICAS DE CONSERVACIÓN } \\
\hline Desconoce & 39,71 & Ninguna & 94 \\
\hline Buena Producción & 10,29 & Cercas Vivas & 1,5 \\
\hline no Produce como antes & 39,71 & Uso de abono Orgánicos & 1,5 \\
\hline necesita Fertilización & 10,29 & Labranza reducida & 1,5 \\
\hline Porcentaje total & 100 & Rotación de cultivos & 1,5 \\
\hline \multicolumn{2}{|l|}{ TIPO DE SUELO } & Porcentaje total & 100 \\
\hline Desconoce & 39,6 & \multicolumn{2}{|c|}{ EROSIÓN } \\
\hline Arcilloso & 47,1 & Desconoce & 39,7 \\
\hline Arenoso & 11,8 & $\mathrm{Si}$ & 35,3 \\
\hline Franco & 1,5 & No & 25 \\
\hline Porcentaje total & 100 & Porcentaje total & $100 \%$ \\
\hline
\end{tabular}

Causas de erosión en las microcuencas de los ríos Cristal y el Salto

El recurso suelo es un medio muy importante dentro de las microcuencas del rio Cristal y El Salto en donde la población alude que según su percepción en lo que respecta a calidad, ellos tienen suelos regulares $(38,3 \%)$, buenos $(13,2 \%)$, malos $(8,8 \%)$ y desconocen en un $39,7 \%$. Lo antes mencionado tiene relación a volúmenes de producción que obtienen los agricultores haciendo referencia a años anteriores. Los tipos de suelo que predominan son: arcillosos, arenosos y francos.

\section{Erosión del recurso suelo}

La población en su mayoría manifiesta el desconocimiento del proceso de erosión de suelos. Sin embargo determinados agricultores indican que sus fincas están degradadas por este fenómeno, y en menor porcentaje revelan que no está erosionado el suelo ya que mantienen prácticas de manejo y conservación con cultivos continuos y/o asociados (perennes). Las causas del proceso antes mencionado son: las lluvias (erosión hídrica), Deforestación y monocultivo (erosión antropogénicas). La aplicación de prácticas de conservación es negativa en un $94 \%$ y apenas un $6 \%$ realizan obras de conservación basadas en cercas vivas, abonos orgánicos, labranza reducida y rotación de cultivos.

\section{Problemas ambientales}

Al consultarles a los responsables de los sistemas de producción establecidos en las microcuencas de los ríos Cristales y el Salto, 
respecto a los principales problemas que afectan a sus sistemas, mencionaron como los más relevantes a los siguientes: degradación de la tierra agrícola y desertificación, deforestación, pérdida de la biodiversidad, contaminación del aire, estrés hídrico, contaminación de los ríos/vertientes, vulnerabilidad ante eventos naturales extremos (sequías), pérdida de la identidad cultural y pobreza de acuerdo a la etnia; sin embargo, para los entrevistados todas los ítems son relevantes demostrando con un porcentaje del $11,1 \%$ por cada una de las opciones que se les ha presentado. Mismos que consideran que los problemas ambientales son muy importantes equivale a un $62,6 \%$, el $9 \%$ consideran que son menos importantes y el $28,4 \%$ no da versión alguna sobre esta problemática. La población considera que el deterioro ambiental está entre moderado/rápido en un 61,3\%, mientras que un 29,4\% desconoce este proceso el $6,4 \%$ piensa que está en detención/reversión y un $3 \%$ de los entrevistados manifiesta que dicho proceso está avanzando muy rápido.

\section{CONCLUSIONES}

En las microcuencas de los ríos Cristal y El Salto, se recopiló la información de 68 familias, luego se analizó y se sistematizo la información que permitió caracterizar los sistemas de producción. Para el análisis de cuencas hidrográficas se requiere de una extensa información de cartografía digitalizada misma que es muy limitada en zonas de alta nubosidad.

Los sistemas de producción identificados y caracterizados en las microcuencas a más de ser deficientes son inequitativos en términos económicos, sociales y ambientales por la poca participación de las Instituciones locales y regionales. Estos componentes condicionan a que sean limitadas las opciones de medios de vida.
Los pobladores de las microcuencas están conscientes de la importancia del ambiente pero sin embargo no realizan ninguna acción de preservación de los recursos naturales. En nuestra investigación se evidenció la poca o casi nada capacitación en alternativas de manejo, preservación y producción sostenible y sustentable de los recursos que poseen.

En las microcuencas las familias se encuentran distribuidas en su gran mayoría al contorno del rio Cristal, porque se encuentra aledaña a la vía principal misma que ven como una oportunidad para generar ingresos económicos. La migración de los pobladores de la microcuenca del rio El Salto se ha dado por la baja productividad agrícola y pecuaria.

Mediante el análisis de los ingresos se determinó que para los pobladores de estas microcuencas, el programa estatal denominado Bono de Desarrollo Humano es prioritario para muy pocas familias porqué la mayoría de ellas tienen ingresos de diversos rubros tales como: Agrícolas, pecuarios, salarios fijos y a contrato, entre otros. El turismo es una nueva actividad que están retomando por la construcción del Parque Acuático Balsapamba, mismo que está potencializando a la zona como un destino turístico.

\section{REFERENCIAS}

Barrera, V. (2006). Estudio de Línea Base Manejo de recursos naturales basado en cuencas hidrográficas en agricultura de pequeña escala: El caso de la subcuenca del río Chimbo, Bolívar, Ecuador, p. 2

Barrera, V.; Cárdenas, F. y Monar, C. (2005). Diagnóstico Participativo con enfoque de género para la subcuenca hidrográfica del río Chimbo. INIAP-SANREMCRSP. Guaranda, Ecuador. $24 \mathrm{pp}$

Barrera V.; León-Velarde, C.; Grijalva, J. y Chamorro, F. (2004). Manejo de sistemas de producción "Papa-Leche" en la sierra Ecuatoriana. INIAP-CIP-PROMSA. Editorial ABYA-YALA. Quito, Ecuador. 196 pp 
Caracterización socioeconómica, ambiental y de género en los sistemas de producción de las microcuencas Ríos Cristal y el Salto - Provincias Bolívar y los Ríos, Ecuador

Cárdenas, F., Barrera, V. (2007). Manejo de recursos naturales basado en cuencas hidrográficas en agricultura de pequeña escala: El caso de la subcuenca del río Chimbo. Diagnóstico Rural Participativo Desde La Equidad De Género, Social Y Ambiental En La Subcuenca Hidrográfica Del Río Chimbo, Provincia De Bolívar, Ecuador. Pp.23-34

González, M. (2008). Caracterización Socioeconómica y Ambiental de los Sistemas de Producción de las Microcuencas del Río Illangama y el Río Alumbre de la Subcuenca del Río Chimbo, provincia Bolívar-Ecuador. Tesis previa a la obtención de Ingeniera Agroforestal de la Universidad Estatal de Bolívar
PEDPB, 2004-2024. Plan Estratégico de Desarrollo Provincial de Bolívar

VI Foro Nacional Climático, (2011). Iniciativas de Adaptación al Cambio Climático en el Sector Agrícola y Recursos Hídricos. Proyecto para la prevención y adaptación al cambio climático y variabilidad climática de la microcuenca Balsapamba Río Cristal, Río El Salto. Gobiernos Provinciales de Los Ríos y Bolívar. Consultado el 22 de Febrero del 2013. En línea:...http://www.portalces.org/index.p hp/index.php?option=com_sobi2\&sobi2T ask=sobi2Details \&sobi2Id=1030\&Itemid= 76 